\title{
Low-speed jet dynamics and sound radiation
}

\author{
André V. G. Cavalieri ${ }^{1 *}$, Daniele Violato ${ }^{2 \dagger}$, Daniel Rodríguez ${ }^{3,4 \ddagger}$, Peter Jordan ${ }^{1 \S}$, \\ Fulvio Scarano $^{2 \uparrow}$, Tim Colonius ${ }^{3 \|}$, Yves Gervais ${ }^{1 * *}$ \\ ${ }^{1}$ Institut Pprime, Poitiers, France \\ ${ }^{2}$ Delft University of Technology, Delft, the Netherlands \\ ${ }^{3}$ California Institute of Technology, Pasadena, CA \\ ${ }^{4}$ Universidad Politécnica de Madrid, Spain
}

\begin{abstract}
Experimental velocity measurements of a low-speed jet, performed using time-resolved tomographic PIV, are used to study the dynamics of large-scale structures and their sound radiation. The experimental results show the roll-up of axisymmetric vortices that pair downstream, and subsequently lose their azimuthal coherence. Models of linear instability waves using both steady laminar and mean-field base flows flow are applied. While good agreement can be obtained for the vortex roll-up frequency in the near-nozzle region using the laminar base flow, non-linear effects must be included, via the mean field, in order to capture the downstream evolution of both the fundamental and subharmonic (vortex pairing). The velocity fluctuations for both frequencies have a wave-packet structure with some jitter in the form of modulations of the spatial extent and amplitude of the envelope. The sound radiation is modelled using a jittering wave-packet model, an shows agreement with the exponential directivity shape of Laufer and Yen (J. Fluid Mech. 134, 1983).
\end{abstract}

\section{Introduction}

Jets at low Reynolds numbers, with laminar boundary layers at the nozzle exit, are of fundamental interest because of their reduced range of turbulent scales. In aeroacoustics this allows the study of the jet dynamics and sound radiation in simplified settings where the flow is dominated by coherent structures. Additionally, it is possible to calculate the flow field using direct numerical simulation.

Experiments performed at low speeds present an additional advantage related to the sampling frequency. Since the frequencies of velocity fluctuations are proportional to $U / D$, low-speed jets allow time-resolved PIV measurements with sampling frequencies corresponding to high Strouhal numbers.

In the present work we analyse the time-resolved tomographic PIV measurements of an unforced water jet by Violato and Scarano ${ }^{1}$ so as to develop models for both the jet dynamics and the radiated sound, as in previous efforts by this group. ${ }^{2-5}$ The lower azimuthal modes are modelled as wavepackets, computed via Parabolised Stability Equations with base flows given either by the jet mean flow or a corresponding laminar velocity profile.

The experiment is performed for a Reynolds number of 5000, and the jet in this case presents a nearperiodic roll-up of vortex rings that subsequently pair and evolve towards an increasingly complex, threedimensional, structure. The velocity fluctuations are measured in a volume with an axial extent of 10 jet diameters. The sampling rate corresponds to a Strouhal number of 20, allowing three-dimensional flow features to be tracked. A comprehensive comparison between the experiment and the proposed models is thereby possible.

\footnotetext{
*PhD student, Institut Pprime, 43 rue de l'Aérodrome, 86036 Poitiers, France.

${ }^{\dagger} \mathrm{PhD}$ student, Delft University of Technology.

$¥$ Marie Curie COFUND fellow, California Institute of Technology.

$\S$ Research Scientist, Institut Pprime, 43 rue de l'Aérodrome, 86036 Poitiers, France.

I Professor, Delft University of Technology.

" Professor, California Institute of Technology.

**Professor, Institut Pprime, 40 avenue du Recteur Pineau, 86022 Poitiers, France.
} 
Although past studies of low-Reynolds number flows suggest that nonlinear interactions play an important role on both the jet dynamics and sound radiation, ${ }^{6-8}$ for high-Re jets good agreement is observed between experiments and fluctuations calculated using a linear PSE, where the experimental mean velocity field is taken as the base flow. ${ }^{3,5}$ In this case non-linearity is implicitly included via the mean flow.

We explore this point by comparing linear PSE solutions computed using both laminar and mean baseflows of the low Reynolds number jet, and by comparing with results from the high Reynolds number, fully turbulent jet studies of Cavalieri et al. ${ }^{9}$ Although the two experiments are not at the same Mach number, the main differences are believed to be mostly due to changes of the Reynolds number and of the boundary layer at the nozzle exit; the present jet is transitional, whereas the high-Re flows are fully turbulent jets. We do not expect linear PSE to fully model the dynamics of vortex pairing in the low-Re jet, but we wish to ascertain to what extent these can be modelled by the implicit nonlinearities included by using the experimental mean flow. In this sense linear PSE is a first step to study the degree of nonlinearity in this transitional jet.

As the present experimental database is of a water jet, no acoustic measurements are available. If we consider that the dynamics of a water jet are not fundamentally different from a low Reynolds, low Mach number air jet (such as studied by Laufer and $\mathrm{Yen}^{10}$ ), we can use the water jet measurements in an aeroacoustic context, and so the acoustic radiation of an equivalent air jet can be calculated using an acoustic analogy. This is done using a jittering wave-packet model, ${ }^{11}$ and reasonable qualitative agreement is found with the experiments of Laufer and Yen. ${ }^{10}$

\section{Experimental apparatus and procedures}

\section{II.A. Jet flow facility}

Experiments were conducted in the water jet facility described by Violato and Scarano ${ }^{12}$ at the Aerodynamic Laboratories of TU Delft in the Aerospace Engineering Department. A round nozzle of exit diameter D=10 $\mathrm{mm}$ and contraction ratio of $56: 1$, was installed at the bottom wall of a tank. The nozzle contraction is shaped following the work of Schram et al. ${ }^{13}$ and yields a uniform laminar flow at the exit. The jet is enclosed in an octagonal water tank of $600 \mathrm{~mm}$ diameter and $800 \mathrm{~mm}$ height, built in Plexiglas to enable extensive optical access for illumination and tomographic imaging (figure 1).

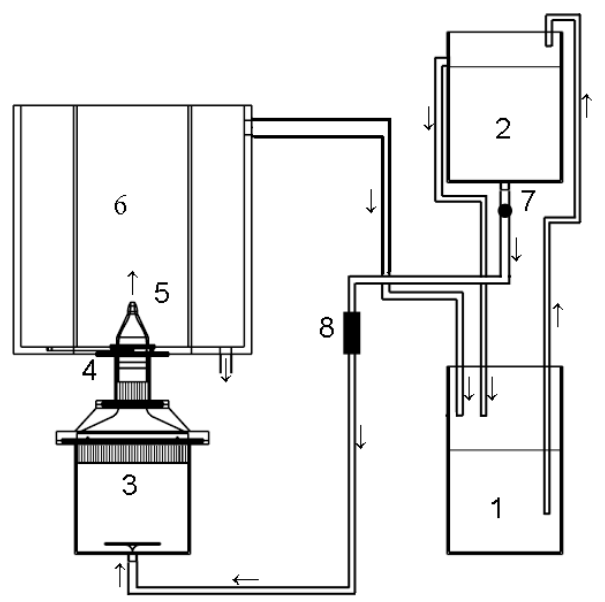

Figure 1. Jet tomographic facility: a) schematic of the system, (1) water reservoir, (2) piezometric water tank, (3) jet tank, (4) settling chamber, (5) nozzle, (6) Plexiglas water tank, (7) valve, (8) flow meter (Violato and Scarano ${ }^{12}$ )

The system is driven hydrostatically and provides a stabilized supply in an exit velocity range $0.1 \mathrm{~m} / \mathrm{s}<$ $U<2 \mathrm{~m} / \mathrm{s}$, corresponding to Reynolds numbers ranging between 1000 and 20000. Tomographic experiments were performed for a nominal axial velocity at the jet exit of $0.5 \mathrm{~m} / \mathrm{s}$, yielding a Reynolds number Re $=5000$ based on the jet diameter $D$.

\section{II.B. Time-resolved tomographic measurements}

Neutrally buoyant polyamide particles of $56 \mu \mathrm{m}$ diameter were dispersed homogeneously, achieving a uniform concentration of 0.65 particles $/ \mathrm{mm}^{3}$. The illumination was provided by a Quantronix Darwin-Duo solid- 
state diode-pumped Nd:YLF laser $(2 \times 25 \mathrm{~mJ} /$ pulse at $1 \mathrm{kHz})$. After a transmission distance of $1.5 \mathrm{~m}$, the laser beam reaches a diameter of $6 \mathrm{~mm}$, is expanded with a concave lens to a diameter of $80 \mathrm{~mm}$ and then focused with a biconvex lens to obtain a conical illumination (figure 2). The light scattered by the particles is recorded by a tomographic system composed of three 3 Imager pro HS 4M cameras arranged horizontally with azimuthal aperture of 90 degrees. The choice of illuminated volume with a beam of circular cross section eliminates the need for a camera-lens tilt mechanism to comply with the Scheimpflug condition. Nikon objectives of $105 \mathrm{~mm}$ focal length were set with a numerical aperture $f_{\#}=22$ to allow focused imaging of the illuminated particles. For the chosen illumination and imaging configuration the particle image density attains a maximum of 0.04 particles/pixel at the jet axis and decreases towards the edge of the illuminated volume. The details of the experimental settings are summarised in table 1.
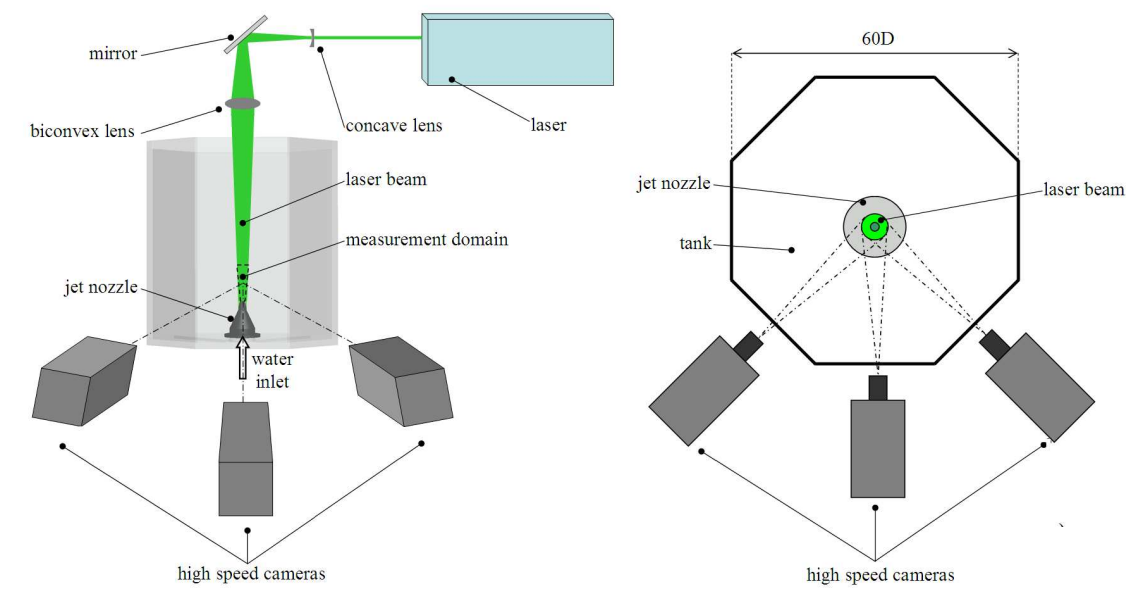

Figure 2. Schematic view of illumination and imaging in the tomographic experiment (left); top view of the system (right).

\begin{tabular}{|l|l|}
\hline Seeding material & $\begin{array}{l}\text { polyamide particles diameter: } 56 \mu \mathrm{m} \\
\text { concentration: } 0.65\left[\text { particles } / \mathrm{mm}^{3}\right]\end{array}$ \\
\hline Illumination & Quantronix Darwin-Duo Nd-YLF laser $(2 \times 25 \mathrm{~mJ} @ 1 \mathrm{kHz})$ \\
\hline Recording device & $\begin{array}{l}3 \text { Imager pro HS } 4 \mathrm{M} \text { cameras } \\
(2016 \mathrm{x} 2016 \text { pixels @ } 1.3 \mathrm{kHz}) \\
11 \mu \text { pixel pitch }\end{array}$ \\
\hline Recording method & $\begin{array}{l}\text { double frame/single exposure } \\
\text { continuous mode }\end{array}$ \\
\hline Optical arrangement & $\begin{array}{l}\text { Nikon objectives }\left(f=105 \mathrm{~mm} ; f_{\#}=22\right) \\
\text { field of view } 5 D \mathrm{x} 10 D\end{array}$ \\
\hline Acquisition frequency & $1 \mathrm{kHz}$ \\
\hline Time of acquisition & $2 \mathrm{~s}$ \\
\hline
\end{tabular}

The choice of a conical domain of illumination also results in a more favorable condition for accurate reconstruction of the particle field, as the particle image density does not change with the viewing angle along the azimuth and decreases moving from the axis to the periphery of the jet (figure 2). Sequences of images of tracer particles were recorded at $1 \mathrm{kHz}$ resulting in a temporal resolution that yields 35 samples for the fastest expected events (vortex shedding). The imaged-particle displacement at the exit is approximately 10 pixels along the jet axis. The field of view is $50 \times 100 \mathrm{~mm}$ with a digital resolution of 18.5 pixels $/ \mathrm{mm}$ and the measurement domain extends as sketched in figure 3 . 


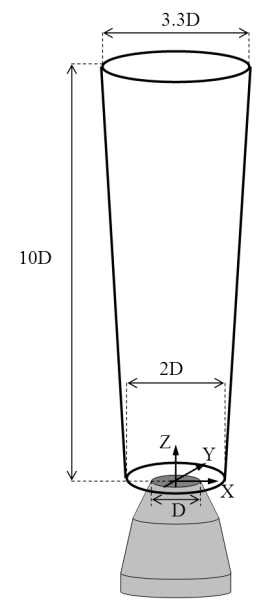

Figure 3. Details of the measurement and coordinate system.

\section{II.C. Tomographic reconstruction}

The volumetric light intensity reconstruction is performed following the Multiplicative Algebraic Reconstruction Technique (MART, Hermann and Lent ${ }^{14}$ ) algorithm by LaVision software Davis 8. A three-dimensional mapping function from image-space to physical object-space is generated by imaging a calibration target. The initial experimental errors due to system calibration are estimated at approximately 0.5 pixels by the disparity vector field (Willert ${ }^{15}$ ). The misalignment is reduced to less than 0.05 pixels making use of the self-calibration technique (Wieneke ${ }^{16}$ ). The raw images are pre-processed with subtraction of the minimum intensity at each pixel for the entire sequence, followed by a subtraction of the local minimum over a kernel of 31x31 pixels. The MART algorithm is applied with four iterations, with a unit value of the pixel-to-voxel ratio.

The illuminated volume is discretised with $900 \times 900 \times 1950$ voxels resulting in a digital resolution of 18.5 voxels $/ \mathrm{mm}$ (voxel pitch of $54 \mu \mathrm{m}$ ). Following Elsinga et al. ${ }^{17}$ the accuracy of the reconstruction is evaluated a-posteriori comparing the intensity of reconstructed particles in the illuminated region with that of ghost particles produced in the immediate surrounding of the illuminated domain. A signal-to-noise ratio may be defined as the reconstructed particle intensity inside the illuminated area versus that reconstructed outside. In the present experiment, the intensity distribution of the laser light is approximately Gaussian and the light is concentrated in the conical volume shown in figure 3 where the signal-to-noise ratio is higher than 2 .

\section{II.D. Vector field computation}

The three-dimensional particle field motion is computed by Volume Deformation Iterative Multigrid (VODIM) technique (Scarano and Poelma ${ }^{18}$ ) with a final interrogation volume of $40 \times 40 \times 40$ voxels $\left(2 \times 2 \times 2 \mathrm{~mm}^{3}\right)$ and an overlap between adjacent interrogation boxes of $75 \%$, leading to a vector pitch of $0.5 \mathrm{~mm}$. At the given particle concentration, 6.5 particles are counted, on average, within the interrogation box.

A further refinement of the analysis is obtained by using a correlation averaging technique whereby the cross-correlation map, obtained over three subsequent object-pairs, is averaged yielding higher signal-to-noise ratio and higher measurement precision. This approach is an extension of the method proposed by Meinhart et al. ${ }^{19}$ here extended to unsteady velocity fields when the sampling rate exceeds that strictly needed for temporally resolving the flow. The use of such an averaging technique involves a longer measurement time interval corresponding to the separation of four exposures $(3 \mathrm{~ms})$.

The relative error in the velocity measurement is approximately $2 \%$ with respect to the particle displacement at the jet exit of 10 voxels.

\section{Instability wave models}

Previous studies ${ }^{3,5}$ have shown good agreement between modes obtained from Linear Parabolised Stability Equations (LPSE) and the fluctuations of pressure and velocity for fully turbulent jets at high Reynolds 
number. The good agreement found between these linear wavepacket models and experiments is attributed, at least partially, to the use of the turbulent mean flow as a base flow in the PSE computations. Some degree of non-linearity is thereby accounted for in the model via the mean field. Nonlinear interactions between the resolved frequency modes are assumed to be small for the unforced turbulent jets studied in these cases. On the other hand, the incompressible, low Reynolds number jet considered here is initially laminar, and an abrupt laminar-turbulent transition occurs around 4-5 diameters downstream of the nozzle. Non-linear effects involving large-scale structures (vortex shedding and pairings) dominate the flow field past the first 1-2 diameters. It is not clear whether these non-linear effects can be captured by a linear PSE model that uses the mean flow field (where zero-frequency non-linear mode interactions are implicit) or whether the non-zero-frequency wave interactions need to be modelled explicitly via a non-linear PSE solver. There is evidence that vortex pairing, such as those observed in the present experiment, can be modelled as nonlinear interactions of instability waves $^{8}$ in a plane mixing-layer similar to the annular mixing-layer. This approach requires the determination of an adequate laminar base flow as input, and which does not correspond to the mean flow.

We explore the above questions by computing solutions of linear PSE using both laminar and mean velocity profiles derived from the experimental data. The solutions are compared both with the measured unsteady velocity data and with the high Reynolds number PSE-experiment comparisons of Cavalieri et al. ${ }^{9}$

\section{III.A. Laminar and mean base flow models}

Two base flows are considered in the PSE computations discussed in what follows. The first is the experimental mean flow, while the second is computed as a solution of the steady laminar boundary-layer equations for axisymmetric flow for the same Reynolds number $R e=5000$ and momentum thickness at the nozzle $\theta / D=0.028$. Figure 4 shows axial velocity contours and velocity profiles for the two base flows. The experimental mean flow spreads much faster than the laminar flow on account of the strong mixing due to the vortical structures and their interactions. The sole mechanism for mixing-layer spreading in the steady laminar case is viscous dissipation, which is too weak to produce such strong changes in the flow on the first few diameters. Figure 4(c) shows the difference between the experimental mean and the steady laminar flow. This difference, which is related to mean flow distortion arising from non-linear interactions, is up to $40 \%$ of the jet velocity.

\section{III.B. PSE results and comparison with experimental data}

The time-resolved, tomographic PIV results allow application of Fourier transforms in time and of a Fourier series in azimuth. Fluctuation amplitudes as a function of frequency $\omega$ and azimuthal wavenumber $m$ can thus be directly compared to PSE results. In what follows we focus on the axisymmetric mode $m=0$, since most of the fluctuations in the first jet diameters are axisymmetric. ${ }^{1}$ Comparisons are made for the two dominant Strouhal numbers identified in this region of the flow, namely the Strouhal number of vortex shedding $(S t=0.7)$ and of vortex pairing $(S t=0.35)$. Figure 5 shows a comparison of the amplitudes on the jet centerline between the experiment and linear PSE results for these two Strouhal numbers.

When the laminar baseflow is used the growth rate of the fundamental frequency $(\mathrm{St}=0.7)$ is captured well, as one would expect, over the first diameter. Downstream of this point the experimental fluctuations saturate, an indication that the non-linear regime has been attained. The non-linearity may be manifest in one of two ways (or indeed by a combination of both): the energy of the fundamental may be transfered to the subharmonic $(\mathrm{St}=0.35)$, and/or a mean-field deformation may be produced which results in a modification of the downstream evolution of the wavepacket. It does not appear possible to capture the evolution of the subharmonic mode using the linear theory and the laminar base flow, an indication that this fluctuation evolves in a nonlinear manner.

When the mean field is used as base flow, and some non-linearity thereby implicitly acknowledged, the evolution of the fundamental mode is captured from $x / D=1$ to $x / D=3$, indicating that the observed modification of the wavepacket evolution can be associated with this change in the mean field. The close agreement of the radial distribution of $\mathrm{St}=0.7$ amplitudes in this region with results of linear PSE, shown in figure 6 , is further evidence of this. Downstream of $x / D=3$ agreement is again poor, as the wavepackets here become overwhelmed by turbulence.

The downstream evolution of the subharmonic mode shows moderate agreement with the linear PSE solution when the mean field is used as the base flow. The less substantial improvement of the agreement 

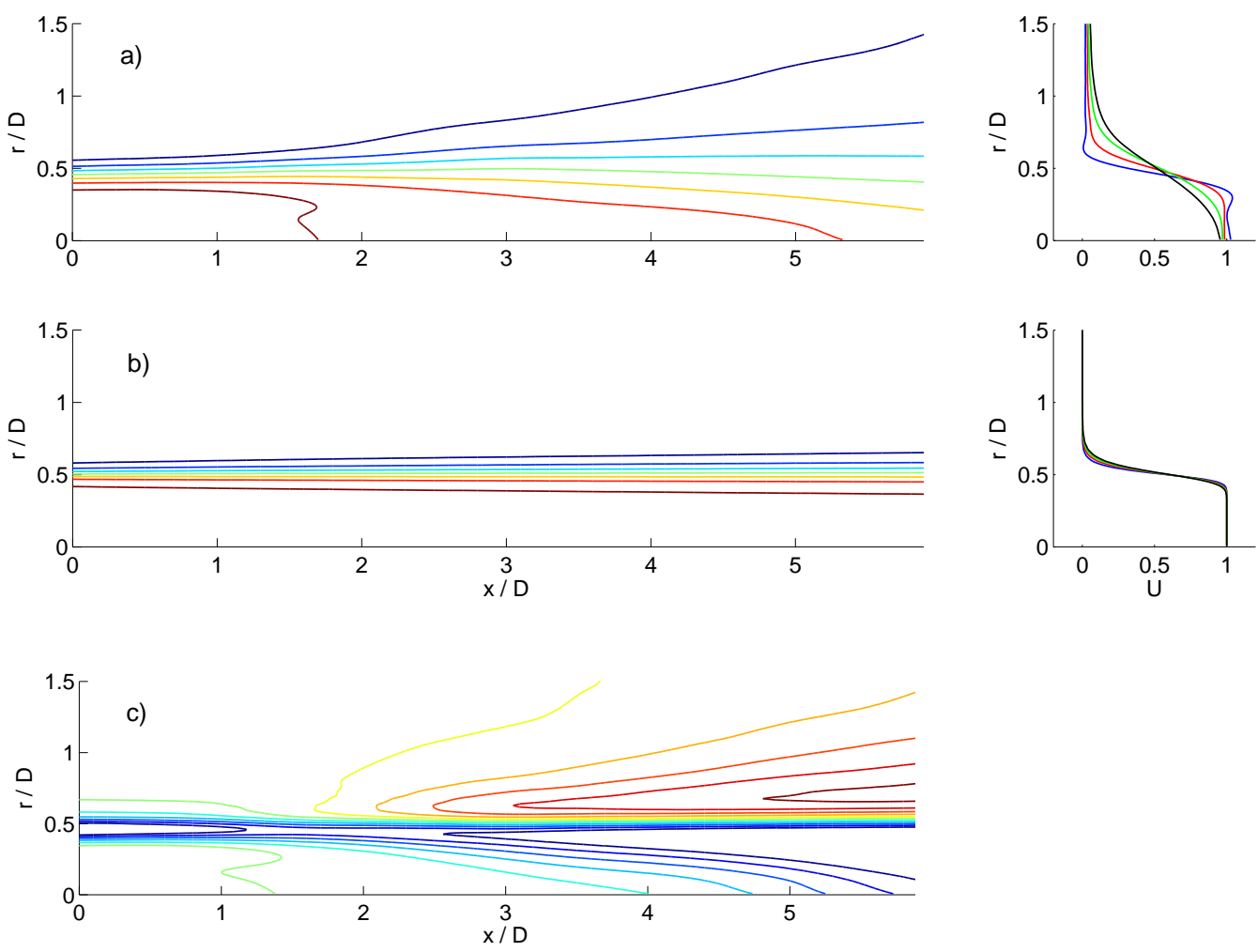

Figure 4. The base flows used in the instability waves model: a) experimental mean flow, b) steady laminar base flow. Left: Contours of axial velocity, in equal increments from 0.1 through 0.99 . Right: Axial velocity profiles at $x / D=0$ (blue), $x / D=1$ (red), $x / D=2$ (black) and $x / D=4$ (green). c) Contours of axial velocity difference between the experimental mean and the steady laminar flow, in equal increments from -0.25 through 0.25 .
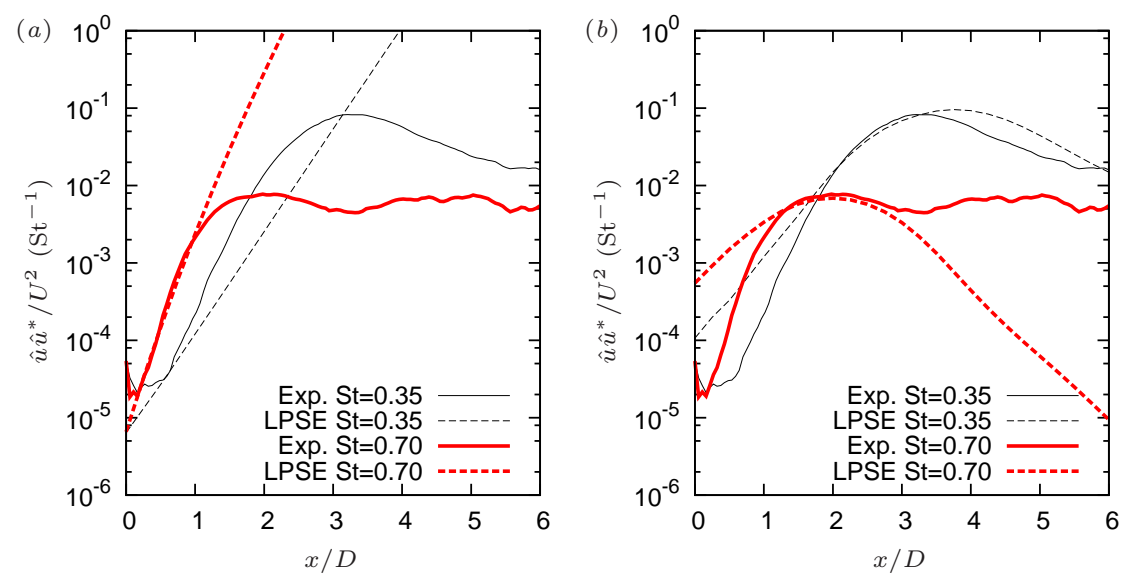

Figure 5. Amplitudes of $u_{x}$ on the jet centerline, compared to linear PSE with base flow given by (a) laminar solution and (b) mean flow

when the mean flow is used suggests that although some nonlinear effects are implicit in the linear PSE calculation, this may not be sufficient to model the growth rate of the subharmonic. Some features of the $\mathrm{St}=0.35$ fluctuations are nonetheless captured by linear PSE, as shown in the radial distribution of amplitudes presented in figure 6; this is reminiscent of the linear PSE results with mean flow correction presented by Cheung and Lele. ${ }^{8}$ The degree to which non-linear mode interactions must be explicitly accounted for can be assessed by means of a non-linear PSE computation, which is currently underway.

Figure 7 compares the high and low Reynolds number cases. The former, taken from Cavalieri et al. ${ }^{9}$ shows comparisons between experimental measurements and linear PSE based on the mean flow of a fully 
$(a)$
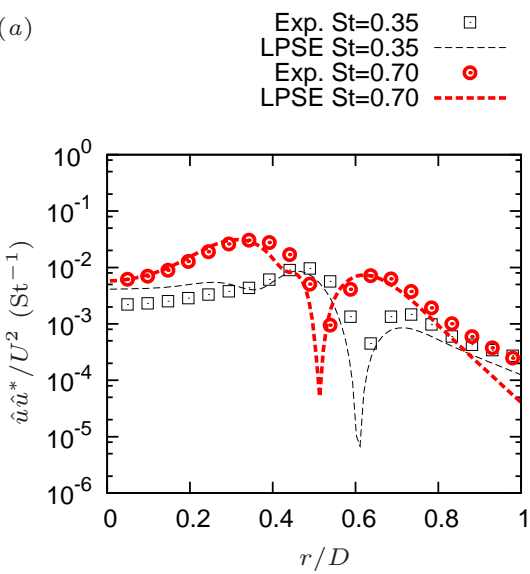

(b)
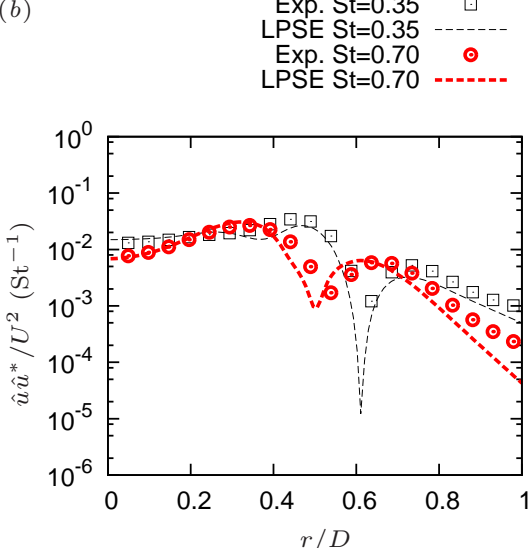

Figure 6. Amplitudes of $u_{x}$ on (a) $x / D=1.5$ and (b) $x / D=2$, compared to linear PSE with base flow given by the mean flow

turbulent, unforced jet, issuing from a nozzle with turbulent boundary layers - which makes clear that the use of a laminar base flow would in this case not be legitimate. Good agreement is observed from the nozzle exit to the end of the potential core, downstream of which, similar to the low Reynolds number case, the wavepackets become overwhelmed by turbulence. We also note the difference in fluctuation amplitude between the high and low Reynolds number cases. The much lower relative levels of the high Re jet in the region upstream of the end of the potential core provide further justification for the use of a linearisation using the mean velocity as base flow.
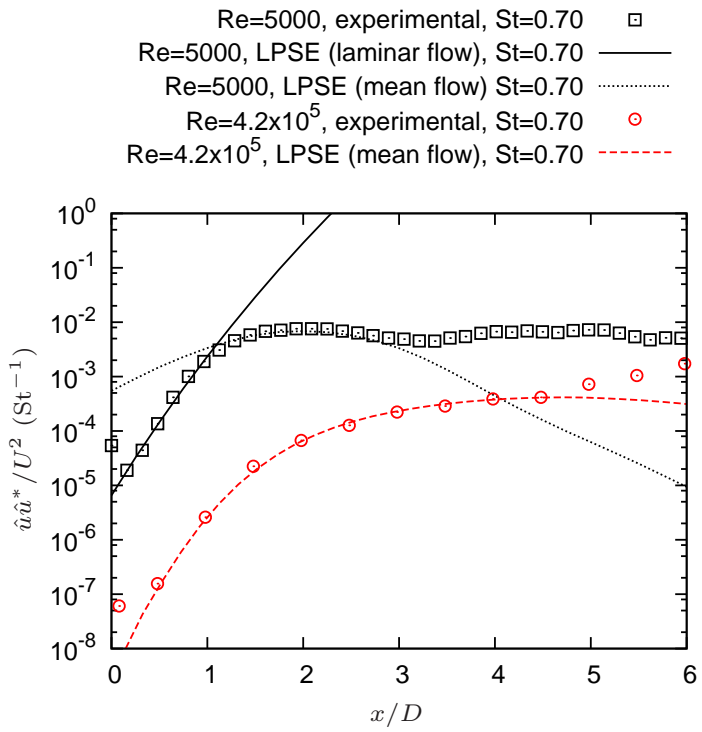

Figure 7. Amplitudes of $u_{x}$ on the jet centerline for the low-Re and high-Re jets, compared to linear PSE.

\section{A model of the sound source for vortex pairing}

To evaluate sound generation by the wave-packet structure of the jet velocity field, analysed in the previous section, we have used the experimental data in Lighthill's acoustic analogy. ${ }^{20}$ As before, we focus here on the axisymmetric mode for both velocity and acoustic fields. 


\section{IV.A. Source decomposition}

To provide comparisons with the experiment of Laufer \& Yen ${ }^{10}$ for air jets, we have performed a calculation based on Lighthill's analogy for an air jet with Mach number of 0.15. We assume that the dynamics of the jet studied are the same as an air jet with the same Reynolds number, both jets being considered as incompressible.

The Strouhal number of the primary instability of the jet is $\mathrm{St}=0.7$. Since we can write

$$
\frac{D}{\lambda}=\operatorname{St} M
$$

for the present values of St and $M$ the assumption of radial compactness is reasonable. ${ }^{11}$ Therefore, to calculate the sound radiation of the axisymmetric mode, we perform a radial integration of the $T_{11}$ component of Lighthill's stresss tensor obtatined experimentally, which is given as

$$
s\left(y_{1}, \tau\right)=2 \pi \rho_{0} \delta(r) \int_{0}^{\infty} U\left(y_{1}, r\right) u\left(y_{1}, r, \tau\right) r \mathrm{~d} r
$$

where $\rho_{0}$ is the density of the fluid, assumed as constant, and $U$ and $u$ are, respectively, the mean value and the fluctuation of the axisymmetric mode of the axial velocity. This integrated source is shown in figure 8( $a)$

(a)

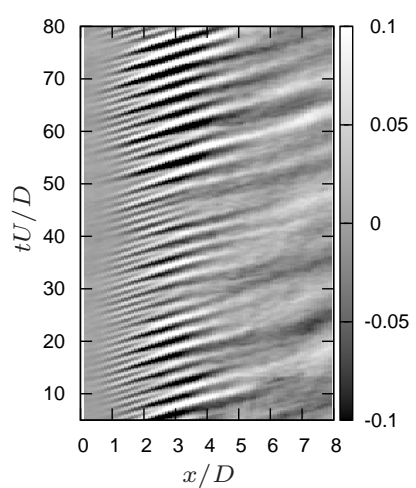

(b)

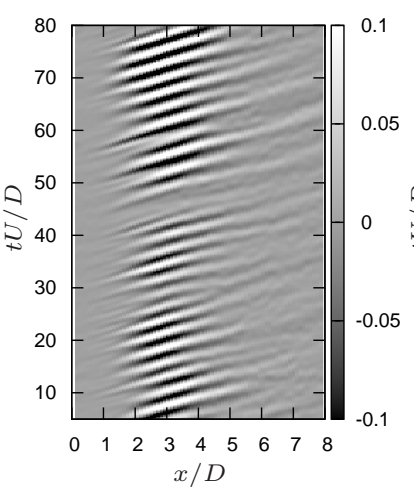

(c)

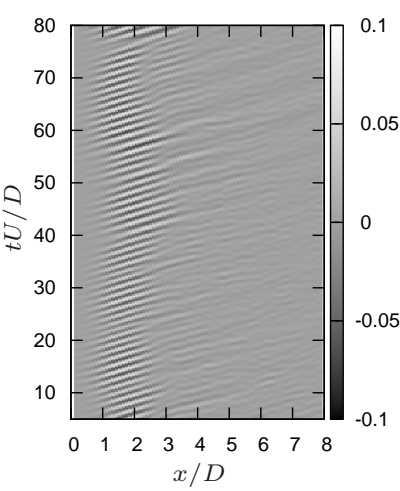

$(d)$

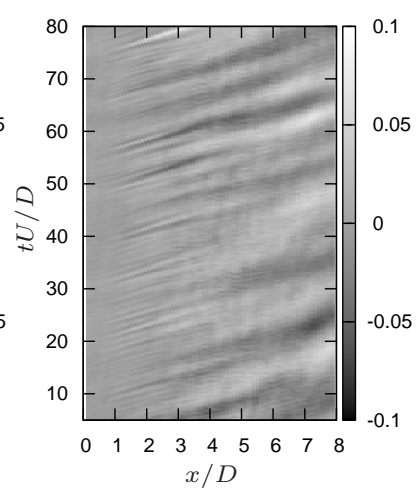

Figure 8. Source decomposition: (a) radially integrated source $s$ of eq. (2); (b) subharmonic and (c) fundamental fluctuations ( $\mathrm{St}=0.35$ and 0.7 , respectively); and (d) residuum.

We see in figure $8(a)$ a source that is similar to a 'jittering' wave-packet, ${ }^{11}$ but with two characteristic frequencies: one related to the vortex roll-up, which corresponds to $\mathrm{St}=0.7$, and one related to the pairing of vortices, at $\mathrm{St}=0.35$. This kind of model, with an envelope corresponding to each frequency, has been suggested by Crigthon \& Huerre ${ }^{21}$ to model the vortex pairing observed by Laufer and Yen. ${ }^{10}$ The approach we pursue here is different in that we account for the jitter in the wave-packet shape. To extract these two wave-packet signatures, we perform a short-time Fourier series, representing the source as the superposition of two harmonics with slowly-changing amplitudes

$$
s\left(y_{1}, t\right)=\sum_{n=1}^{2}\left[A_{n}\left(y_{1}, t\right) \cos (n \omega t)+B_{n}\left(y_{1}, t\right) \sin (n \omega t)\right]
$$

where the coefficients are calculated by

$$
\begin{aligned}
& A_{n}\left(y_{1}, t\right)=\frac{2}{T} \int_{t-T / 2}^{t+T / 2} s\left(y_{1}, \tau\right) \cos (n \omega \tau) \mathrm{d} \tau \\
& B_{n}\left(y_{1}, t\right)=\frac{2}{T} \int_{t-T / 2}^{t+T / 2} s\left(y_{1}, \tau\right) \sin (n \omega \tau) \mathrm{d} \tau,
\end{aligned}
$$

and $\omega$ is taken as the frequency of the subharmonic. The source field is then split into three parts: a quasiharmonic oscillation at $\omega$, related to the region downstream of the pairing; a quasi-harmonic oscillation at 
$2 \omega$, related to the vortex roll-up; and a residuum. These three parts of the source are shown, respectively, in figs. $8(b),(c)$ and $(d)$ for a calculation with $T$ taken as the oscillation period of the subharmonic, $2 \pi / \omega$. We see in figures $8(b)$ and $(c)$ that both the subharmonic and the fundamental have the shape of a jittering wave-packet: a hydrodynamic wave representing the convection of structures, with temporal variations of both amplitude and spatial extent of the wavepacket envelope. We will focus on these two parts of the source in what follows, neglecting the residuum shown in figure $8(d)$.

\section{IV.B. Parameters of the jittering wave-packet}

Due to the observed wave-packet shape of the source, we can fit it with an analytical equation corresponding to a wave with Gaussian modulation

$$
s_{n}(\mathbf{y}, \tau)=\delta\left(y_{2}\right) \delta\left(y_{3}\right) \mathcal{A}_{n}(\tau) \mathrm{e}^{\mathrm{i}\left(n \omega \tau-k_{n} y_{1}\right)} \mathrm{e}^{-\frac{y_{1}^{2}}{L_{n}^{2}(\tau)}},
$$

where we allow temporal variations of the amplitude $\mathcal{A}_{n}$ and of the spatial extent $L_{n}$ for each of the two principal frequencies.

To calculate the instantaneous values of $\mathcal{A}_{n}$ and $L_{n}$, we have fitted Gaussians at each timestep for the envelope $E_{n}\left(y_{1}, \tau\right)$, which is calculated for each frequency as $\sqrt{A_{n}\left(y_{1}, \tau\right)^{2}+B_{n}\left(y_{1}, \tau\right)^{2}}$. Some sample fits for the fundamental and the subharmonic are shown respectively in figures 9 and 10. We note that the Gaussian function accurately represents the shape of the instantaneous envelopes for both frequencies.
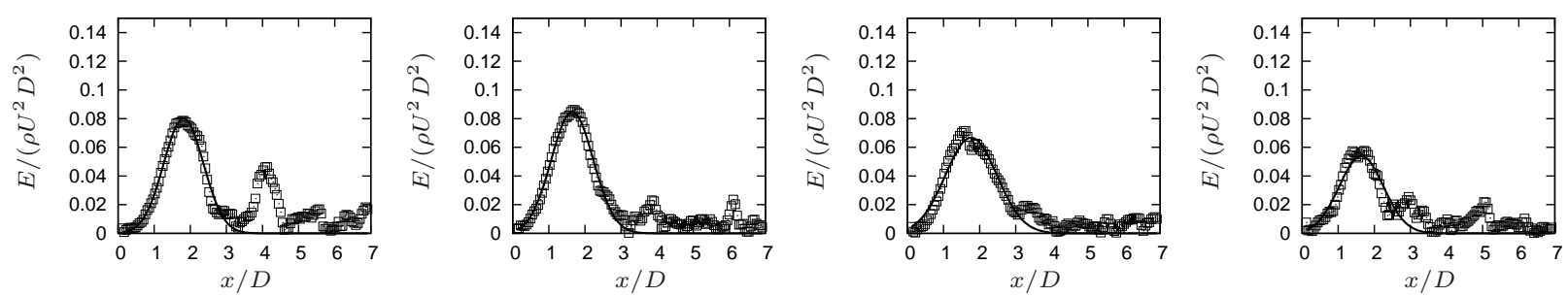

Figure 9. Envelopes for the fundamental frequency at (a) $t U / D=8.35,(b) t U / D=16.70,(c) t U / D=25.05$ and (d) $t U / D=33.40$. Symbols show the calculated envelope $E\left(y_{1}, \tau\right)$, and lines show Gaussian fits.
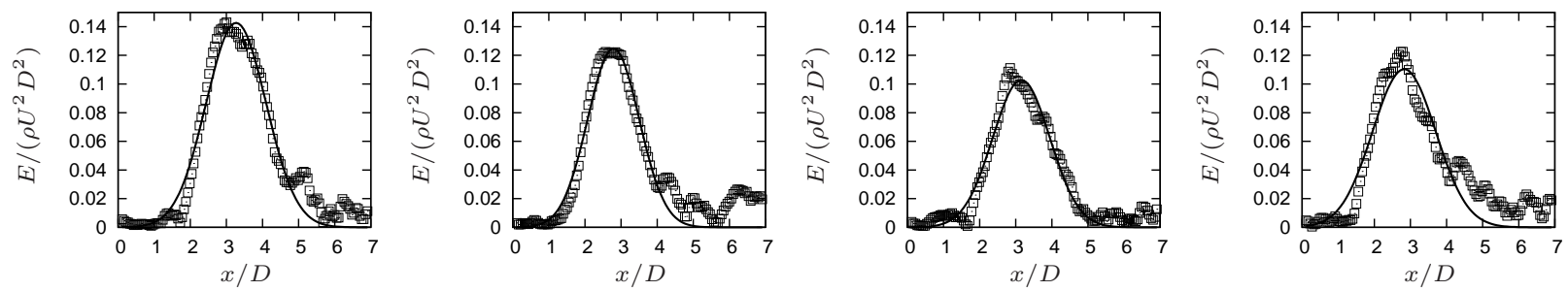

Figure 10. Envelopes for the subharmonic frequency at $(a) t U / D=8.35,(b) t U / D=16.70,(c) t U / D=25.05$ and (d) $t U / D=33.40$. Symbols show the calculated envelope $E\left(y_{1}, \tau\right)$, and lines show Gaussian fits.

The average convection velocity, calculated as $\omega / k$, was determined using the peaks in the frequencywavenumber spectrum, leading to $0.542 U$ for the fundamental and $0.6 U$ for the subharmonic.

\section{Acoustic radiation}

The radiated sound field is given for Lighthill's analogy as

$$
p(\mathbf{x}, t)=\frac{1}{4 \pi} \iiint\left[\frac{1}{|\mathbf{x}-\mathbf{y}|} \frac{\partial^{2} T_{i j}}{\partial y_{i} \partial y_{j}}(\mathbf{y}, \tau)\right]_{\tau=t-\frac{|\mathbf{x}-\mathbf{y}|}{c}} \mathrm{~d} \mathbf{y}
$$

which, for the present simplified source, is given in the far acoustic field as ${ }^{11}$

$$
p(\mathbf{x}, t)=-\mathcal{A}^{*} \frac{\rho_{0} U^{2} M_{c}^{2}(k D)^{2} L^{*} \sqrt{\pi} \cos ^{2} \theta}{8|\mathbf{x}|} \mathrm{e}^{-\frac{\left(L^{*}\right)^{2} k^{2}\left(1-M_{c} \cos \theta\right)^{2}}{4}} \mathrm{e}^{\mathrm{i} \omega\left(t-\frac{|\mathbf{x}|}{c}\right)},
$$


where $^{*}$ refers to values taken at a retarded time $\tau=t-x / c$.

The calculated directivities for the fundamental and the subharmonic are plotted in figure 11. For this calculation we have considered the parameters of Laufer and Yen's experiment. ${ }^{10}$ Figure $11(a)$ shows that the subharmonic radiation has a much higher amplitude than the fundamental, although the directivity patterns are similar. Higher amplitudes for the subharmonic are also found in the experiment of Bridges \& Hussain, ${ }^{22}$ but the differences in amplitude are lower than in the present case. From the analysis of section III we can say that the non-linear dynamics underpin the stronger radiation of the subharmonic, but that this non-linearity is at least partially captured when the mean velocity profile is used in a linearisation of the Navier-Stokes equations.
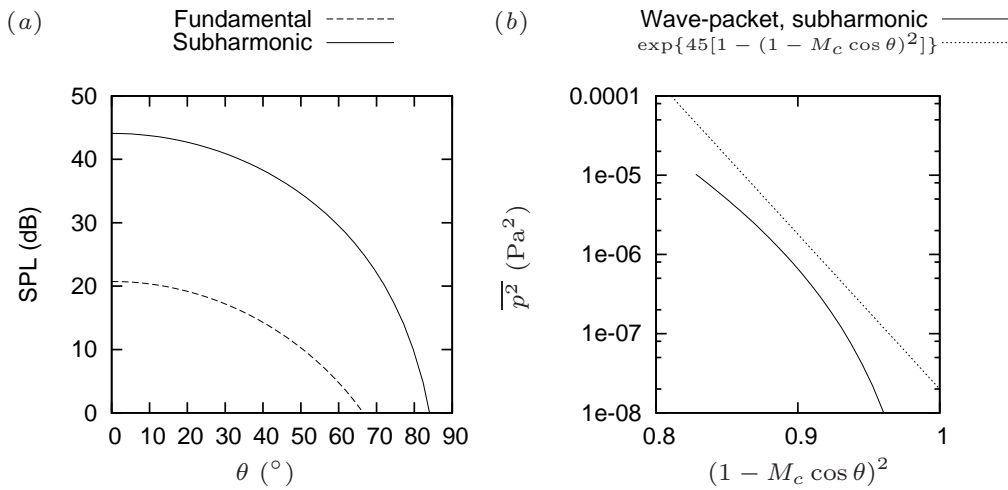

Figure 11. Wave-packet results: (a) directivity for fundamental and subharmonic wave-packets, and (b) comparison of subharmonic directivity with Laufer and Yen's directivity shape

Figure 11(b) presents a comparison of the calculated directivity for the subharmonic with the exponential directivity obtained experimentally by Laufer and Yen for forced jets. The calculated directivity for the present experiment is close to the expression $\exp \left\{45\left[1-\left(1-M_{c} \cos \theta\right)^{2}\right]\right\}$, but with a slightly lower exponential decay. In Laufer and Yen's experiment the decay was indeed lower for unforced jets, and this has been attributted to the temporal variations of the wave-packet, which are considered in the present model.

\section{Conclusion and perspectives}

We have used velocity measurements obtained by time-resolved tomographic PIV applied to a low-speed jet to study its dynamics and sound radiation. Preliminary comparisons between the measured velocity fluctuations and wavepackets computed using Parabolised Stability Equations show that in the near-nozzle region the initial stages of the fundamental, roll-up, frequency can be understood as a linear wave growing on a laminar base flow. This saturates downstream of $x / D=1$, at which point its evolution can only be captured by considering non-linear effects via a deformation of the mean field. A linear PSE computation using the mean field allows the evolution of the wavepacket to be correctly captured up to $x / D=3$, after which transition to turbulence occurs. The subharmonic cannot be understood in the same way. In the near-nozzle region neither of the computations correctly capture its evolution, while downstream of $x / D=2$ better agreement can be obtained if, again, non-linear effects are accounted for by using the mean velocity field as a base flow rather than the laminar field. Further improvements may be possible by the explicit modelling of non-linear wave interactions using a non-linear PSE solver.

Comparison of the above behaviour with results from a high Reynolds number study (where the fluctuation amplitudes are an order of magnitude lower) reinforce the contention that a good deal of the nonlinearity of fully turbulent jets can be accounted for in linear models for wavepacket dynamics, provided the linearisation is performed about the mean velocity field.

The velocity fluctuations are furthermore seen to present a wave-packet structure with a certain amount of jitter, and the associated sound radiation has been modelled using simplified analytical models. ${ }^{11}$ The sound propagation for low axial angles was calculated using such a model, and the directivity shape agrees with the experimental results of Laufer and Yen. ${ }^{10}$ 


\section{Acknowledgments}

The authors kindly acknowledge LaVision GmbH and Piero Colonna for providing the CMOS cameras used in the TOMO-PIV experiments.

This work was conducted as part of the FLOVIST project (Flow Visualization Inspired Aeroacoustics with Time Resolved Tomographic Particle Image Velocimetry), funded by the European Research Council (ERC), grant no 202887. This present work was partially supported by CNPq, National Council of Scientific and Technological Development - Brazil, and through the EU-Russian program ORINOCO (FP7-AAT-2010RTD-Russia; project number 266103).

\section{References}

${ }^{1}$ Violato, D. and Scarano, F., "Three-dimensional evolution of flow structures in transitional circular and chevron jets," Physics of Fluids, Vol. 23, No. 12, 2011, pp. 124104-124104.

${ }^{2}$ Suzuki, T. and Colonius, T., "Instability waves in a subsonic round jet detected using a near-field phased microphone array," Journal of Fluid Mechanics, Vol. 565, 2006, pp. 197-226.

${ }^{3}$ Gudmundsson, K. and Colonius, T., "Instability wave models for the near-field fluctuations of turbulent jets," Journal of Fluid Mechanics, Vol. 689, 2011, pp. 97-128.

${ }^{4}$ Colonius, T., Samanta, A., and Gudmundsson, K., "Parabolized stability equation models of large-scale jet mixing noise," IUTAM Symposium on Computational Aero-Acoustics for Aircraft Noise Prediction, Southampton, UK, March 29-31 2010.

${ }^{5}$ Rodríguez, D., Samanta, A., Cavalieri, A. V. G., Colonius, T., and Jordan, P., "Parabolized stability equation models for predicting large-scale mixing noise of turbulent round jets," 17th AIAA/CEAS Aeroacoustics Conference and Exhibit, Portland, OR, USA, June 5-8 2011.

${ }^{6}$ Mohseni, K., Colonius, T., and Freund, J. B., "An evaluation of linear instability waves as sources of sound in a supersonic turbulent jet," Physics of Fluids, Vol. 14, 2002, pp. 3593.

${ }^{7}$ Sandham, N. D. and Salgado, A. M., "Nonlinear interaction model of subsonic jet noise," Philosophical Transactions of the Royal Society A, Vol. 366, No. 1876, 2008, pp. 2745-2760.

${ }^{8}$ Cheung, L. C. and Lele, S. K., "Linear and nonlinear processes in two-dimensional mixing layer dynamics and sound radiation," Journal of Fluid Mechanics, Vol. 625, 2009, pp. 321.

${ }^{9}$ Cavalieri, A. V. G., Rodríguez, D., Jordan, P., Colonius, T., and Gervais, Y., "Wavepackets in the velocity field of turbulent jets," 18th AIAA/CEAS Aeroacoustics Conference and Exhibit, Colorado Springs, CO, USA, June 4-6 2012.

${ }^{10}$ Laufer, J. and Yen, T.-C., "Noise generation by a low-Mach-number jet," Journal of Fluid Mechanics, Vol. 134, 1983, pp. $1-31$.

${ }^{11}$ Cavalieri, A. V. G., Jordan, P., Agarwal, A., and Gervais, Y., "Jittering wave-packet models for subsonic jet noise," Journal of Sound and Vibration, Vol. 330, No. 18-19, 2011, pp. 4474-4492.

${ }^{12}$ Violato, D. and Scarano, F., "Three-dimensional evolution of flow structures in transitional circular and chevron jets," to appear in Physics of Fluids, 2011.

${ }^{13}$ Schram, C., Taubitz, S., Anthoine, J., and Hirschberg, A., "Theoretical/empirical prediction and measurement of the sound produced by vortex pairing in a low Mach number jet," Journal of Sound and Vibration, Vol. 281, No. 1-2, 2005, pp. $171-187$.

${ }^{14}$ Herman, G. and Lent, A., "Iterative reconstruction algorithms," Computers in Biology and Medicine, Vol. 6, No. 4, 1976, pp. $273-294$

${ }^{15}$ Willert, C., "Stereoscopic digital particle image velocimetry for application in wind tunnel flows," Measurement science and technology, Vol. 8, 1997, pp. 1465.

${ }^{16}$ Wieneke, B., "Volume self-calibration for 3D particle image velocimetry," Experiments in Fluids, Vol. 45, No. 4, 2008, pp. $549-556$

${ }^{17}$ Elsinga, G. E., Scarano, F., Wieneke, B., and Van Oudheusden, B. W., "Tomographic particle image velocimetry," Experiments in Fluids, Vol. 41, No. 6, 2006, pp. 933-947.

${ }^{18}$ Scarano, F. and Poelma, C., "Three-dimensional vorticity patterns of cylinder wakes," Experiments in Fluids, Vol. 47, No. 1, 2009, pp. 69-83.

${ }^{19}$ Meinhart, C. D., Wereley, S. T., and Santiago, J. G., "A PIV algorithm for estimating time-averaged velocity fields," Journal of Fluids Engineering, Vol. 122, No. 2, 2000, pp. 285-289.

${ }^{20}$ Lighthill, M. J., "On sound generated aerodynamically. I. General theory," Proceedings of the Royal Society of London. Series A, Mathematical and Physical Sciences, 1952, pp. 564-587.

${ }^{21}$ Crighton, D. G. and Huerre, P., "Shear-layer pressure fluctuations and superdirective acoustic sources," Journal of Fluid Mechanics, Vol. 220, 1990, pp. 355-368.

${ }^{22}$ Bridges, J. E. and Hussain, A. K. M. F., "Roles of initial condition and vortex pairing in jet noise," Journal of Sound and Vibration, Vol. 117, No. 2, 1987, pp. 289-311. 